\title{
Neurodegeneration: accelerated ageing or inadequate healthcare?
}

\author{
Olga Golubnitschaja
}

Published online: 29 June 2010

(C) European Association for Predictive, Preventive and Personalised Medicine 2010

\begin{abstract}
Current figures: About 300 million Diabetics frequently affected by Poly-Neuropathy as secondary complication, 18 million patients with Alzheimer's disease also diagnosed as Diabetes Type 3, neurodegenerative eye diseases with leading causes of blindness - diabetic retinopathy and estimated 67 million glaucoma patients worldwide, millions of patients with Parkinson's disease, Multiple Sclerosis, Epilepsy, Cerebral Palsy and Dementia in the elderly - altogether dramatically affect life quality, social and economical indexes of populations around the globe. Optimistic versus Pessimistic Prognosis depends much on diagnostic, preventive and treatment approaches which healthcare will preferably adopt in the near future. Without innovation in healthcare, neurodegenerative disorders can reach more than $30 \%$ of global disease burden till 2020. In contrast, effective utilisation of advanced early/ predictive diagnostics, preventive and personalised medical approaches could enable a significant portion of population to reach the 100-year age limit remaining vibrant in excellent physical and mental health as actively contributing members of society.
\end{abstract}

Keywords Neurodegeneration $\cdot$ Ageing $\cdot$ Healthcare . Early/predictive diagnosis - Targeted preventive measures . Personalised medicine

\footnotetext{
O. Golubnitschaja $(\bowtie)$

Department of Radiology,

Rheinische Friedrich-Wilhelms-University of Bonn,

Sigmund-Freud-Str. 25,

53105 Bonn, Germany

e-mail: Olga.Golubnitschaja@ukb.uni-bonn.de

O. Golubnitschaja

European Association for Predictive,

Preventive \& Personalised Medicine,

Brussels, Belgium
}

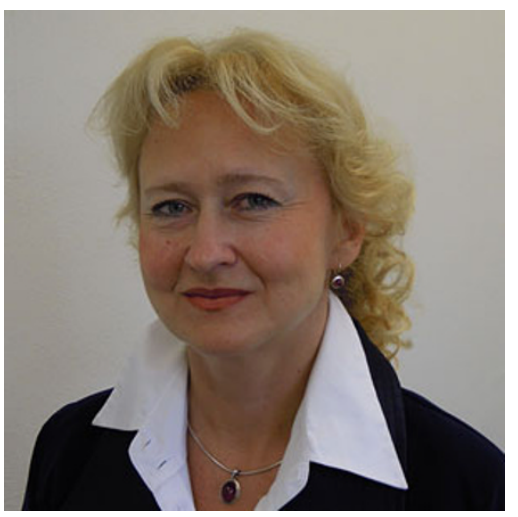

Olga Golubnitschaja, Editor-in-chief, the EPMA Journal

\section{Neurodegeneration as the common feature of multifactorial physical and cognitive disability}

Progressive neurodegeneration is the common feature of the majority of socially and economically devastating disorders and diseases with multifactorial physical and cognitive disability. Permanently increasing numbers of those patients as well as dramatic disease-related economical burden indicate that current education, research and healthcare in this field are far from optimal and should be corrected. Since neurodegeneration is a chronic process, which begins long before the onset of clinically diagnosed symptoms, currently there is an evident lack of targeted prevention in any age-category inchoate with early childhood, over adolescence till late adulthood. This is supported by current age-related dramatic increase in numbers of patients with any kind of neurodegenerative medical condition.

\section{When should we start to protect individuals against neurodegeneration?}

As soon as possible! Thus, the task of individual prediction and well-timed targeted prevention of the life-long chronic 
pathologies e.g. developed by newborns particularly affected by mild or severe asphyxia should be given the extraordinary priority in paediatrics. Birth asphyxia is the most frequent perinatal complication [1]. Secondary to birth asphyxia, a postnatal manifestation of hypoxic-ischemic encephalopathy is frequently observed being associated with either mild or severe organ damage in asphyxiated newborns, both leading to the development of chronic pathologies including progressive neuro/degenerative processes, CNS-injury, epilepsy, schizophrenia, psychotic syndromes, diabetes mellitus, vascular pathologies, nephropathy, cardiomyopathy, cancer, senescence [2]. From viewpoint of innovative perinatal/ postnatal diagnostic tools to prognose pathology development and individual outcomes, there is a great potential behind new prenatal/neonatal animal models of progressive neurodegeneration as reviewed in this journal-issue by A. Tasker et al. [3]. Those models enable identifying targets in predictive diagnostics and pre-symptomatic treatment with relevance to epilepsy, schizophrenia, amyotrophic lateral sclerosis, Parkinson's and other neurodegenerative diseases, and open good perspectives for targeted prevention early in life.

\section{Neurodegenerative eye-diseases as indicator for chronic medical conditions: can we protectively act in advance?}

The idea of "A Sick Eye in a Sick Body" has been formulated by F. Lagrange in 1922 [4] and still counts as being valid and supported for several pathologies by the most advanced diagnostic and follow-up technologies as reviewed by M. Pache \& J. Flammer [5]. Thus, proliferative diabetic retinopathy is attracting much attention counted for the leading cause of blindness worldwide and considered as an early indicator for individual predisposition to the cascade of chronic complications responsible for the majority of morbidity and mortality in diabetics. Chronic degenerative processes are well-known as the severe complication secondary to diabetes; the overall pathomechanisms work as the "domino-effect" in a characteristic sequence of events beginning with the "up-stream" manifestation of proliferative retinopathy followed by the cascade of neurodegenerative processes in further organs as reviewed earlier $[6,7]$. The overall update to the topic has been recently performed by the EPMA-Journal with the specialised issue dedicated to the "Predictive, Preventive \& Personalised Medicine in Diabetes" in the 1st quarter of 2010. Several reviews demonstrated the primary focus on proliferative retinopathy before the global neurodegenerative cascade is triggered, in favour of early/predictive diagnosis, targeted preventive measures and indications for individualised treatment approaches. This should build the reliable platform for advanced diabetes care as preventive and personalised medicine to effective costs [8-15].
Worldwide the second leading cause of blindness with nearly 8 million people bilaterally blind among altogether 65 million patients is the glaucomatous optic neuropathy characterised by a chronic progressive loss of retinal ganglion cells and their neurons. As reviewed in this journal-issue, the clue to preventing blindness from glaucoma is early/predictive diagnosis and effective personalised treatment of predisposed individuals [16, 17]. Moreover, accelerated ageing triggered by an imbalanced implication of oxidative stress seems to a have a high impact for the disease progression. Consequently, if applied early enough, a protection against oxidative stress e.g. at the level of mitochondrial (dys)function effectively reduces the overall glaucoma risk and can be achieved by dietary supplements such as gingko, dark chocolate, polyphenolic flavonoids that is sufficiently present in green tea, coffee and red wine, as discussed by M. Mozaffarieh et al. [16].

Age-related macular degeneration (AMD) results in visual deficits because of damage to the central retina. Worldwide about 30 million people mainly after 50 years of age are affected by the disease. Macular degeneration counts among multifactorial diseases; current treatment focuses on late stages of the pathology being expensive and less effective approach. To the best acknowledged risk factors of AMD belong genetic background and increased (oxidative) stress such as smoking and unfavourable / toxic environment leading to accelerated ageing of predisposed individuals. As reviewed by P. Hasler [18] in this journal-issue, accurately diagnosed disease's risk and progression as well as targeted personalised treatment approaches (potential targets and corresponding preventive approaches are discussed in the review-article) would ultimately delay or even prevent AMD in the elderly.

Completely new vision about the origin and pathomechanisms of a retinal vein occlusion (RVO) is presented in this journal-issue by the group of Flammer, Department of Ophthalmology, University of Basel [19]. Several clinical observations such as the low impact of anticoagulation or thrombocytopenia on RVO, spontaneous reversibility in young patients and the positive effect of anti-VEGF therapy led the authors to the new concept of pathology namely the local venous spasm triggered by vasoconstrictive messenger-molecules which are released by hypoxia-affected tissues. As published earlier, ocular ischemia resulting from blood-flow deficits may play a major role also in the initiation of glaucoma [20]. In consensus, comparative subcellular imaging and "gene hunting"-investigations performed ex vivo with blood samples of healthy vasospastic individuals versus glaucoma patients and controls provide molecular basis and evidence for vasospasm as predisposition to glaucoma [21]. Moreover, a potential to develop further related (neuro)degenerative pathologies has not been excluded by 
the authors. Therefore, primary vascular deregulation (Vasospastic Syndrome) seems to be an important risk factor for several (neuro)degenerative diseases as review by $\mathrm{K}$. Yeghiazaryan et al. in this journal-issue [22]. Before the manifest neurodegeneration, a predictive molecularprofiling ex vivo performed in blood-samples of healthy vasospastic individuals can specify individual predisposition followed by the most effective form of protection, namely targeted prevention.

\section{Alzheimer's disease is one of the global challenges of predictive, preventive and personalised medicine in 21 st century}

Current prognosis for the prevalence of Alzheimer's Disease (AD) is depressing: about 110 million patients by 2025 or even more depending on demographic growth of the elderly around the globe. Concomitant social and economical burden are threatening as well documented by experts elsewhere. Therefore, current philosophy behind curative medicine and healthcare of the elderly should be essentially reconsidered in favour of preventive strategies triggered early in life, preselection of predisposed individuals by predictive diagnostic approaches followed by targeted individualised treatments. Further, assistive technologies should be well developed to cover current deficits in effective supervision of already diseases people.

Due to the extensive demographic profile of ageing populations and the multifaceted task of dementia treatment, the whole spectrum of aspects around predictive diagnostics, targeted prevention and personalised treatment of Alzheimer's patients is discussed particularly detailed in this journal-issue. As reviewed by S. Mandel et al. [23], both neurodegenerative $\mathrm{AD}$ and Parkinson's Disease (PD) are considered to be progressive disorders of multifactorial origin with a long preclinical period that ensures the great impact of predictive biomarkers to diagnose predisposed individuals before the manifest pathology, to discriminate between neurodegenerative pathologies with similar clinical symptoms, to provide a measure of disease progression, and to evaluate individual drug efficacy. As reviewed by I. Grossman et al. [24], the $\mathrm{AD}$-dedicated research programmes are essential to validate currently available biomarkers for their clinical utility in supporting targeted preventive measures, paving the road for rational health management and ensuring innovative reimbursement programmes developed by policy-makers. In terms of targeted neuroprotection, current particular interest is focused on several forms of taupathies and TAU-protein related drugs to break the potentially toxic aggregates, inhibiting phosphorylation, accelerating dephosphorylation of TAU-protein and related microtubule interaction as reviewed by I. Gozes [25].
Moving toward a predictive and personalised clinical approach in multiple and amyotrophic lateral sclerosis

Multiple Sclerosis (MS) is a chronic late-onset autoimmune disease with multifactorial aetiology and obvious interplay of genetic, epigenetic and environmental risk factors. MS is affecting preferably young females being clinically characterised by manifestation of accumulating symptoms such as numbness, vertigo, depression, motor weakness and incoordination, urinary retention, visual deficits, pain attacks, heart intolerance, cognitive dysfunction and others as reviewed by I. Grossman \& A. Miller [26]. Curative treatments for MS are currently unavailable. Reduction of relapses' frequency is provided by the injection of immunosuppressants, however, with limited individual efficacy within the patients' cohort. MS intervention therapy is associated with safety limitations and lack of a reliable measure for drug efficacy / treatment response. As concluded by the authors, extensive efforts of international consortia are essential in the field to consolidate the accumulated expertise and create robust diagnostic approaches and targeted individualised treatment of MS patients [26].

Amyotrophic lateral sclerosis (ALS) is the next impressive example to illustrate current absence of predictive diagnostic tools and preventive measures to preselect and protect predisposed individuals against rare neurodegenerative diseases with cognitive/motor disability and fatal outcomes. The outstanding review prepared by B. Nefussy \& V. Drory [27] discusses the whole spectrum of the ALS problematic clearly demonstrating inadequate healthcare management and deficits in multidisciplinary performance to care for this patients' cohort. Thus, the prevalence of over $90 \%$ of sporadic cases indicates the multifactorial character of the disease with currently unclear pathogenesis and individual risk factors. As frequently has been noted here for the above listed neurodegenerative diseases, also in the case of ALS an oxidative stress seems to play the central role triggering the pathology and worsening outcomes. In consensus, mitochondrial dysfunction and genetic deficits in the central detoxification pathway based on $\mathrm{Cu}-\mathrm{Zn}$ superoxide-dismutase 1 activity have been reported for familial ALS cases. Therefore, several hypothetic stress factors leading to accelerated ageing and, further supported by genetic predisposition should be taken into consideration at the molecular level to select diseasespecific targets, in order to develop powerful diagnostic tools for preselection and effective preventive measures for ALS-predisposed individuals. Certain efforts have been undertaken to cover the above mentioned deficits, particularly to improve current treatment in the field. As reviewed by C. Benkler et al. [28] new therapies consider possibly a multi-drug character of treatment as the most 
promising approach: innovative multifunctional therapeutic strategies in ALS are focused on neuroprotective approaches, CNS-related repairing gene-therapy, targeted replacements by stem-cells and others.

\section{Concluding remarks}

- Multifactorial origin of the absolute majority of neurodegenerative disorders and diseases is evident: generally there is an inter-play of genetic, epigenetic and environmental risk factors with resulting imbalanced (oxidative) stress as the clue to pathomechanisms.

- Because of particular functional sensitivity of retinal cells towards stress signals, neurodegenerative eyediseases play a central role in early/predictive diagnostics of degenerative process; therefore, the task to develop reliable predictive tools in ophthalmology should be given the extraordinary priority.

- Neurodegenerative processes are accelerated by dominant stress factors and ageing that can and should be individually recognised basically at molecular level to create the most effective targeted protective strategies and individualised treatment before manifest pathology-the reliable platform for personalised approaches in prevention of neurodegenerative medical conditions.

- Particular accents should be put to primary prevention: preselection of predisposed individuals early in life followed by targeted preventive measures should be well regulated by innovative reimbursement programmes developed by policy-makers. This is considered as preventive medicine of future to effective costs.

- Multifunctional therapeutic strategies or so-called "cocktail" therapies should be developed for single multifactorial pathologies to improved inadequate treatment.

- Extensive efforts of international consortia are essential in the field to consolidate the accumulated expertise and to create robust diagnostic approaches, targeted individualised treatment and effective prevention.

- Utilisation of advanced early/predictive diagnostics, preventive and personalised medical approaches could enable a significant portion of population to reach the 100 -year age limit remaining vibrant in excellent physical and mental health as actively contributing members of society.

\section{References}

1. Peeva V, Golubnitschaja O. Birth asphyxia as the most frequent perinatal complication. In: Golubnitschaja O, editor. Predictive diagnostics and personalized treatment: dream or reality? New York: Nova Science; 2009. p. 499-507.
2. Yeghiazaryan K, Peeva V, Morelli M, et al. Potential targets for early diagnosis and neuroprotection in asphyxiated newborns. In: Golubnitschaja $\mathrm{O}$, editor. Predictive diagnostics and personalized treatment: dream or reality? New York: Nova Science; 2009. p. 509-25.

3. Tasker A, Adams-Mariott AL, Shaw Ch. New animal models of progressive neurodegeneration: tools for identifying targets in predictive diagnostics and presymptomatic treatment. EPMA-J. 2010. doi:10.1007/s13167-010-0019-0.

4. Lagrange F. Du glaucoma et de Ihypotonie; leur traitement vhirurgical. Paris: Librairie Octave Doin; 1922.

5. Pache M, Flammer J. A sick eye in a sick body? Systemic findings in patients with primary open-angle glaucoma. Surv Ophthalmol. 2006;51:179-211.

6. Golubnitschaja $\mathrm{O}$. Clinical proteomics in application to predictive diagnostics and personalized treatment of diabetic patients. Current Proteomics. 2008;5:35-44.

7. Golubnitschaja O. Advanced diabetes care: three levels of prediction, prevention \& personalized treatment. Curr Diabetes Rev. 2010;6:42-51.

8. Golubnitschaja O. Time for new guidelines in advanced diabetes care: paradigm change from delayed-interventional to predictive, preventive \& personalised medicine. EPMA-J. 2010;1:3-12.

9. Abebe W, Mozaffari MS. Endothelial dysfunction in diabetes: potential application of circulating markers for advanced diagnostic and prognostic tools. EPMA-J. 2010;1:32-45.

10. Al-Shabrawey M, Smith S. Prediction of diabetic retinopathy: role of oxidative stress and relevance of apoptotic biomarkers. EPMA-J. 2010;1:56-72.

11. Josifova T, Henrich PB, Pleastina-Borjan I. Proliferative diabetic retinopathy: predictive and preventive measures at hypoxia induced retinal changes. EPMA-J. 2010;1:73-7.

12. Zenoni S, Comi N, Fontana P. Individualized treatment of proliferative diabetic retinopathy: optimal surgical timing improves long-term outcomes. EPMA-J. 2010;1:78-81.

13. Schrader WF, Josifova T. The options to minimize the surgical trauma to treat ocular diabetic complications and to improve postoperative recovery and quality of life require an individualized approach. EPMA-J. 2010;1:82-7.

14. Li Calzi S, Neu M, Shaw LC, Grant M. Endothelial progenitor dysfunction in the pathogenesis of diabetic retinopathy: treatment concept to correct diabetes-associated deficits. EPMA-J. 2010;1:88-100.

15. Sena CM, Bento CF, Pereira P, Seica R. Diabetes mellitus: new challenges innovative therapies. EPMA-J. 2010;1:138-63.

16. Mozaffarieh M, Fraenkl SA, Konieczka K, Flammer J. Targeted preventive measures and advanced approaches in personalised treatment of glaucoma neuropathy. EPMA-J. 2010. doi:10.1007/ s13167-010-0018-1.

17. Golubnitschaja O, Yeghiazaryan K, Flammer J. Key molecular pathways affected by glaucoma pathology: is predictive diagnosis possible? EPMA-J. 2010. doi:10.1007/s13167-010-0031-4.

18. Hasler P, Flammer J. Predictive, preventive and personalised medicine for age-related macular degeneration. EPMA-J. 2010. doi:10.1007/s13167-010-0017-2.

19. Fraenkl S, Mozaffarieh M, Flammer J. Retinal vein occlusions: the potential impact of a dysregulation of the retinal veins. EPMA-J. 2010. doi:10.1007/s13167-010-0025-2.

20. Flammer J, Orgül S, Costa VP, Orzalesi N, Krieglstein GK, Serra LM, et al. The impact of ocular blood flow in glaucoma. Prog Retin Eye Res. 2002;21:359-93.

21. Yeghiazaryan K, Flammer J, Orgül S, Wunderlich K, Golubnitschaja O. Vasospastic individuals demonstrate significant similarity to glaucoma patients as revealed by gene expression profiling in circulating leukocytes. Mol Vision. 2009;15:2339-48.

22. Yeghiazaryan K, Flammer J, Golubnitschaja O. Predictive molecular profiling in blood of healthy vasospastic individuals: 
clue to targeted prevention as personalised medicine to effective costs. EPMA-J. 2010. doi:10.1007/s13167-010-0032-3.

23. Mandel S, Morelli M, Halperin I, Korczyn AD. Biomarkers for prediction and targeted prevention of Alzheimer's and Parkinson's diseases: evaluation of drug clinical efficacy. EPMA-J. 2010; doi:10.1007/s13167-010-0036-Z

24. Grossman I, Lutz MW, Crenshaw D, et al. Alzheimer's disease: diagnostics, prognostics and the road to prevention. EPMA-J. 2010. doi:10.1007/s13167-010-0024-3.

25. Gozes I. Tau pathology: predictive diagnostics, targeted preventive and personalized medicine and application of advanced research in medical practice. EPMA-J. 2010. doi:10.1007/s13167-010-0029-y.
26. Grossman I, Miller A. Multiple sclerosis parmacogenetics: personalized approach towards tailored therapeutics. EPMA-J. 2010. doi:10.1007/s13167-010-0020-7.

27. Nefussy B, Drory VE. Moving toward a predictive and personalized clinical approach in amyotrophic lateral sclerosis: novel developments and future directions in diagnosis, genetics, pathogenesis and therapies. EPMA-J. 2010. doi:10.1007/ s13167-010-0027-0.

28. Benkler C, Offen D, Melamed E, et al. Recent advances in amyotrophic lateral sclerosis research: perspectives for personalized clinical application. EPMA-J. 2010. doi:10.1007/s13167010-0026-1. 